\title{
Subgrid-scale model based on the vorticity gradient tensor for rotating turbulent flows
}

\author{
Han $\mathrm{Qi}^{1,2} \cdot$ Xinliang $\mathrm{Li}^{1,2} \cdot$ Changping $\mathrm{Yu}^{1}$
}

Received: 8 October 2019 / Revised: 5 February 2020 / Accepted: 4 March 2020 / Published online: 3 June 2020

(c) The Chinese Society of Theoretical and Applied Mechanics and Springer-Verlag GmbH Germany, part of Springer Nature 2020

\begin{abstract}
A new subgrid-scale (SGS) stress model is proposed for rotating turbulent flows, and the new model is based on the traceless symmetric part of the square of the velocity gradient tensor and the symmetric part of the vorticity gradient tensor (or the so-called vorticity strain rate tensor). The new subgrid-scale stress model is taken into account the effect of the vortex motions in turbulence, which is reflected on the anti-symmetric part of the velocity gradient tensor. In addition, the eddy viscosity of the new model reproduces the proper scaling as $O\left(y^{3}\right)$ near the wall. Then, the new SGS model is applied in large-eddy simulation of the spanwise rotating turbulent channel flow. Different simulating cases are selected to test the new model. The results demonstrate that the present model can well predict the mean velocity profiles, the turbulence intensities, and the rotating turbulence structures. In addition, it needs no a second filter, and is convenient to be used in the engineering rotational flows.
\end{abstract}

\section{Graphic abstract}

Keywords Large-eddy simulation $\cdot$ Subgrid-scale model $\cdot$ Rotating turbulent channel flow

\section{Introduction}

Rotational turbulence is an important phenomenon in natural and engineering flows, especially in geophysics, astrophysics and turbines. Now, large-eddy simulation (LES) approach, which calculates the large-scale fluid motions directly from the filtered Navier-Stokes equations with modeled the unresolved motions, has made great progresses in simulation of turbulent flows.

So far, there are several different SGS models proposed for large-eddy simulation. The Smagorinsky model is the first subgrid-scale (SGS) stress model proposed by Smagorinsky [1] (1963) and Deaedorff [2]. It is the most widely used viscosity model due to its robustness and simplicity in form. Based on the eddy-damped quasi-normal Markovian theory, Chollet and Lesieur [3] suggested the spectral

Changping $\mathrm{Yu}$

cpyu@imech.ac.cn

1 LHD, Institute of Mechanics, Chinese Academy of Sciences, Beijing 100190, China

2 School of Engineering Science, University of Chinese Academy of Sciences, Beijing 100049, China eddy-viscosity model, which is suitable for homogeneous and isotropic turbulence. Cui et al. [4] proposed a new subgrid eddy-viscosity model which was formulated directly from the filtered Navier-Stokes equation. It performs well in rotating turbulence and wall-bounded turbulent flows. Vreman [5] also gave eddy-viscosity model (Vreman model) which is able to adequately handle not only turbulence but also transitional flow. Nicoud et al. [6] proposed another eddy-viscosity model ( $\sigma$-model) derived from the analysis of the singular values of the resolved velocity gradient tensor. The $\sigma$-model has better results than the dynamic model with a low computational cost. Recently, Yu et al. [7] supplied a new eddy-viscosity model, where the eddy viscosity is closely associated with the correlation between the large-scale strain rate tensor and vorticity gradient tensor. The model (RSM) can predict both the energy and helicity spectra better than the traditional eddy-viscosity model, and it can also obtain reasonable predicting result in wall turbulence and compressible flows. In addition, there are some important LES methods, such as the dynamic procedure. The famous dynamic procedure was proposed by Germano et al. [8], using the Germano identity to determine the coefficient of the SGS model dynamically in LES of turbulent flows. Lilly [9], Piomelli [10], and Meneveau et al. [11] 
improved and generalized the dynamic procedure, promoting the dynamic procedure to become the most commonly used method in LES of turbulence. With the Germano identity, $\mathrm{Yu}$ et al. [12] derived an expression of the subgrid-scale dissipation at the test-filter scale, which can be adopted to optimize the coefficient of SGS models.

Nevertheless, large-eddy simulation of rotating turbulence has not get so good results by using traditional SGS models. To investigate the rotating effects of turbulence, Tafti and Vanka [13] firstly used the Smagorinsky model to simulate the spanwise rotating channel flow. Vreman model and sigma model were also applied to simulate spanwise rotating channel flow by Jiang et al. [14], but they didn't obtain rather good results on the mean velocity profiles and the turbulence intensity. A subgrid-scale model based on coherent structures proposed by Hiromichi et al. [15] was applied in rotating homogeneous turbulence and turbulent channel flow. This model obtained almost the same results as those from the dynamic Smagorinsky model. Yang et al. [16] applied a modified nonlinear subgrid-scale model (MDNSM) for large-eddy simulation of rotating turbulent channel flows. The modified nonlinear model predicts superior results to the previous nonlinear models. Then Yang et al. [17] proposed a new dynamic global-coefficient nonlinear subgrid-scale model (DNVM-G) for large-eddy simulation of rotating turbulent channel flow. The model can get good results in some cases and can successfully predicts the major structures in rotating turbulent channel flow, but the model is rather complicated, which makes the new model difficult to use. Maurits et al. [18] also proposed a nonlinear subgrid-scale model for large-eddy simulation of rotating turbulent flows. The suggested model can behave as well as the dynamic Smagorinsky model without requiring (dynamic) adaptation.

Although some above models contain antisymmetric part of deformation tensor, which may get good results in rotating flows, the influence of vorticity gradient tensor has not been taken into account. Recently, Teitelbaum et al. [19] showed that the helicity has a major impact on the decay rate and can strengthen the local inverse energy cascade when rotation is presented. At the same time, many facts [7] have revealed that the influence of helicity should be considered in subgrid-scale model.

The present model based on the symmetric part of vorticity gradient tensor, considers the contributions of the helicity to the subgrid-scale model. The new model has $O\left(y^{3}\right)$ behavior in the vicinity of the wall and satisfies a correct asymptotic behavior to a wall for incompressible flows. In the following parts, we will present the derivation of the new model, test cases and discussion.

\section{Governing equations and subgrid scale model}

\subsection{Governing equations}

In incompressible rotating turbulent flows, the LES governing equations can be obtained by filtering the Navier-Stokes equations with the low-pass filter,

$\frac{\partial \bar{u}_{i}}{\partial x_{i}}=0$,

$\frac{\partial \bar{u}_{i}}{\partial t}+\frac{\partial \bar{u}_{i} \bar{u}_{j}}{\partial x_{j}}=-\frac{1}{\rho} \frac{\partial \bar{p}}{\partial x_{i}}+v \frac{\partial^{2} \bar{u}_{i}}{\partial x_{j} \partial x_{j}}+2 \varepsilon_{i j k} \bar{u}_{j} \Omega_{k}-\frac{\partial \tau_{i j}}{\partial x_{j}}$,

where $\bar{u}_{i}$ is the filtered velocity, $\rho$ is the density, $\bar{p}$ is the filtered pressure, $v$ is the kinematic viscosity, $\Omega_{k}$ is the rotation component. $\tau_{i j}$ is the SGS stress tensor,

$\tau_{i j}=\overline{u_{i} u_{j}}-\bar{u}_{i} \bar{u}_{j}$,

which should be modeled. The eddy-viscosity model is chosen here, and it can be written as

$\tau_{i j}-\frac{1}{3} \tau_{k k} \delta_{i j}=-2 v_{s g s} \bar{S}_{i j}$,

where $\bar{S}_{i j}=1 / 2\left(\partial_{j} \bar{u}_{i}+\partial_{i} \bar{u}_{j}\right)$ is the resolved (velocity) strain rate tensor, and $\nu_{s g s}$ is the SGS viscosity which needs to be modeled.

In the Smagorinsky model (SM), the SGS viscosity is proposed as

$v_{s g s}=\left(C_{s} \Delta\right)^{2} \sqrt{2 \bar{S}_{i j} \bar{S}_{i j}}$

The constant $C_{s}$ in $\mathrm{SM}$ is given as 0.18 .

RSM model is obtained from the resolved helicity transportation equation, the energy and helicity spectra. The modeled subgrid-scale stress is

$\tau_{i j}^{\bmod }=-2 C_{r} \Delta^{5 / 2} \bar{S}_{S R} \bar{S}_{i j}$

where

$\bar{S}_{S R}=\left|2 \bar{S}_{i j} \bar{R}_{i j}\right|^{1 / 2}$,

$C_{r}=\left(\frac{2}{3}\right)^{\frac{3}{2}} \pi^{-\frac{5}{2}}\left(\alpha C_{K} C_{H}^{2}\right)^{-\frac{1}{2}}$.

$\bar{R}_{i j}$ is the resolved vorticity strain rate tensor, $C_{K}$ and $C_{H}$ can be prescribed a priori or empirically. For example, it is suggested that $C_{K}=1.6[7]$ and $C_{H}=1.0[7,20]$. 


\subsection{Construction of the subgrid-scale model}

One of the aims of the new model is to adapt to the wall. The new model based on the resolved vorticity gradient tensor (VOM) reproduces the proper scaling at the wall $\left(v_{s g s}=O\left(y^{3}\right)\right)$.

$\partial \bar{u}_{i} / \partial \bar{x}_{i}=0$ can be obtained from continuity equation and the resolved velocity can be expanded in the limit $y \rightarrow 0$, as follows:

$\bar{u}_{x}=\left.\frac{\partial \bar{u}_{x}}{\partial y}\right|_{y=0} y+\left.\frac{\partial^{2} \bar{u}_{x}}{\partial y^{2}}\right|_{y=0} y^{2}+O\left(y^{3}\right)$,

$\bar{u}_{y}=\left.\frac{\partial^{2} \bar{u}_{y}}{\partial y^{2}}\right|_{y=0} y^{2}+O\left(y^{3}\right)$

$\bar{u}_{z}=\left.\frac{\partial \bar{u}_{z}}{\partial y}\right|_{y=0} y+\left.\frac{\partial^{2} \bar{u}_{z}}{\partial y^{2}}\right|_{y=0} y^{2}+O\left(y^{3}\right)$.

Obviously, $\partial \bar{u}_{x} /\left.\partial y\right|_{y=0}, \partial^{2} \bar{u}_{x} /\left.\partial y^{2}\right|_{y=0}, \partial^{2} \bar{u}_{y} /\left.\partial y^{2}\right|_{y=0}$, $\partial \bar{u}_{z} /\left.\partial y\right|_{y=0}, \partial^{2} \bar{u}_{z} /\left.\partial y^{2}\right|_{y=0}$ are functions of space and time, but are not related to $y$. It can be shown that $\bar{R}_{i j} \bar{S}_{i j}$ and $\bar{S}_{i j} \bar{R}_{i j}$ are of order $O(1)$. A simple way to get a better behaviour is to remodel the eddy-viscosity.

Firstly, the traceless symmetric part of the square of the velocity gradient tensor can be considered

$S_{i j}^{t}=\frac{1}{2}\left(\bar{L}_{i j}^{2}+\bar{L}_{j i}^{2}\right)-\frac{1}{3} \delta_{i j} \bar{L}_{k k}^{2}$.

By using $\boldsymbol{\Omega}_{i j}$ as the anti-symmetric part of $L_{i j}$ (the velocity gradient tensor $\left.\partial u_{i} / \partial x_{j}\right), S_{i j}^{t}$ can be rewritten as:

$S_{i j}^{t}=\bar{S}_{i k} \bar{S}_{k j}+\overline{\boldsymbol{\Omega}}_{i k} \overline{\boldsymbol{\Omega}}_{k j}-\frac{1}{3} \delta_{i j}\left[\bar{S}_{m n} \bar{S}_{m n}-\overline{\boldsymbol{\Omega}}_{m n} \overline{\boldsymbol{\Omega}}_{m n}\right]$.

Secondly, it can be shown that $S_{i j}^{t} \bar{R}_{i j}$ behaves like $O(y)$. Thus $S_{i j}^{t} \bar{R}_{i j}$ can be rewritten as $\left(S_{i j}^{t} \bar{R}_{i j}\right)^{3}$ which behaves like $O\left(y^{3}\right)$ near the wall.

Thirdly, the new eddy-viscosity should have the same dimension with traditional eddy-viscosity, and thus $\Delta^{5}\left(S_{i j}^{t} \bar{R}_{i j}\right)^{3} /\left(\bar{S}_{i j} \bar{S}_{i j}\right)^{4}$ can be considered. However, there is chance of zero in $\left(\bar{S}_{i j} \bar{S}_{i j}\right)^{4}$, and $\left(S_{i j}^{t} S_{i j}^{t}\right)^{2}$ must be considered to get no divergence in the eddy-viscosity.

Finally, a new local eddy-viscosity $\nu_{s g s}$ can be obtained as:

$v_{s g s}=\left(C_{n} \Delta\right)^{5} \frac{\left(S_{i j}^{t} \bar{R}_{i j}\right)^{3}}{\left(\bar{S}_{i j} \bar{S}_{i j}\right)^{4}+\left(S_{i j}^{t} S_{i j}^{t}\right)^{2}}$.
Assuming that the VOM model gives the same ensemble-average subgrid kinetic energy dissipation as the RSM model, the constant $C_{n}$ is obtained:

$C_{n}^{5}=\frac{C_{r}}{\Delta^{2.5}} \frac{\left\langle\sqrt{2}\left(\bar{S}_{i j} \bar{R}_{i j}\right)^{3 / 2}\right\rangle}{\left\langle\bar{S}_{i j} \bar{R}_{i j} \overline{O P}_{1} / \overline{O P}_{2}\right\rangle}$,

where $\overline{O P}_{1}=\left(S_{i j}^{t} \bar{R}_{i j}\right)^{3}, \overline{O P}_{2}=\left(\bar{S}_{i j} \bar{S}_{i j}\right)^{4}+\left(S_{i j}^{t} S_{i j}^{t}\right)^{2}$.

As has been shown in Eq. (8), the constant $C_{r}$ is determined by $C_{K}, C_{H}$ and $\alpha$. $\alpha$ can be chosen as $k^{-1}(\Delta / \pi)$ or a value between 0 and $1 . C_{n}$ can be assessed numerically using several fields of homogeneous isotropic turbulence. The value of $C_{n}$ can be obtained in the range $0.05 \leqq C_{n} \leqq 0.15$. The model coefficient $C_{n}$ is set to 0.1 in the following part. As the eddy-viscosity is scaled as $O\left(y^{3}\right)$, it tends to be zero in near-wall region and laminar region where it should be zero theoretically. As discussed by Yu et al. [7], $\bar{S}_{i j} \bar{R}_{i j}$ reflects the intensity of the local helicity flux, and the $S_{i j}^{t} \bar{R}_{i j}$ can also reflect the influences of the local helicity flux to the subgridscale stress model. As suggested by Johnston [21], the flow is destabilized in one part of the channel and stabilized in the other part by the Coriolis force. The unstable part is named pressure side and the stable part is named suction side. The suction side will tend to laminarization as the Rossby number increases [22-24]. And the eddy viscosity of the VOM will tend to zero in the suction part, especially at high Rossby number.

\section{Numerical method and discussion}

The Fourier-Chebyshev pseudo-spectral algorithm method is applied to solve the LES governing equations. The second-order Adams-Bashforth scheme is applied to the nonlinear term for temporal integration. The dealiasing error is removed by the phase-shift method together with the $3 / 2$ rule. The new subgrid-scale model is applied to spanwise rotating turbulent channel flows, whose computational domain is three dimensional box of size $4 \pi h \times 2 h \times 2 \pi h$ ( $h$ is the half-width of the channel). The schematic of the computational domain is plotted in Fig. 1. Different Reynolds numbers $\left(R e_{\tau}=u_{\tau} h / \nu\right.$, where $u_{\tau}$ is the friction velocity, $h$ is the channel half-width and $\nu$ is the kinematic viscosity) and rotation numbers $\left(R o_{\tau}=2 \Omega h / u_{\tau}\right.$, where $\Omega$ is the angular velocity) are chosen in the simulation cases. The four sets of cases are $R e_{\tau}=180, R o_{\tau}=22 ; R e_{\tau}=180$, $R o_{\tau}=40 ; R e_{\tau}=180, R o_{\tau}=80 ; R e_{\tau}=435, R o_{\tau}=10.3$ differently, and named as case 1 , case 2 , case 3 and case 4 . In case 1 , the grid resolution $32 \times 33 \times 32$ is used. In case 2 , 


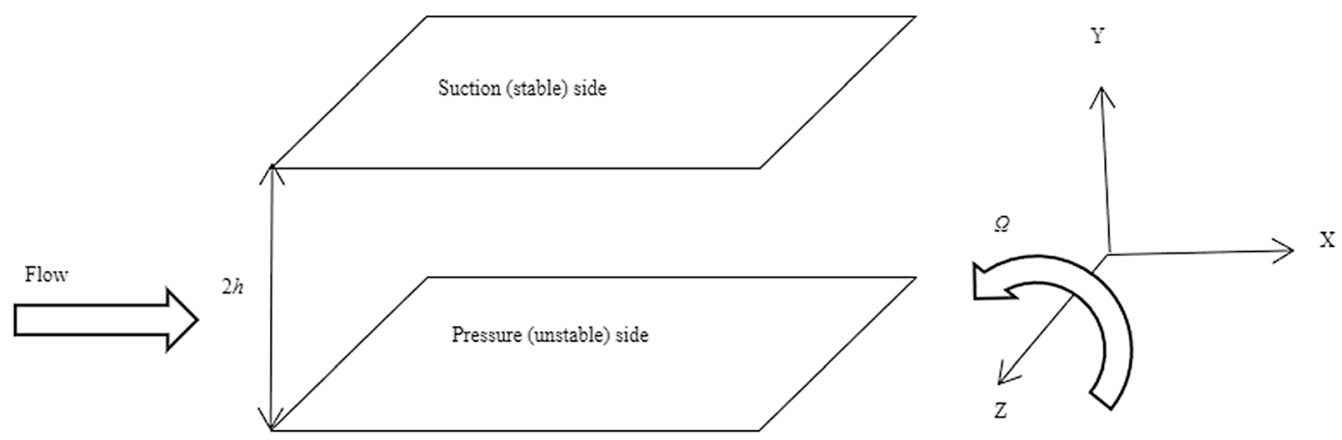

Fig. 1 Schematic of the geometry $(h=1)$

the grid resolution $32 \times 49 \times 32$ is used. In case 3 , the grid resolution $32 \times 57 \times 32$ is used. In case 4 , the grid resolution $64 \times 65 \times 64$ is used. The results can be seen in Sect. 4 . And the analyses of the grid independence can be seen in Appendix.
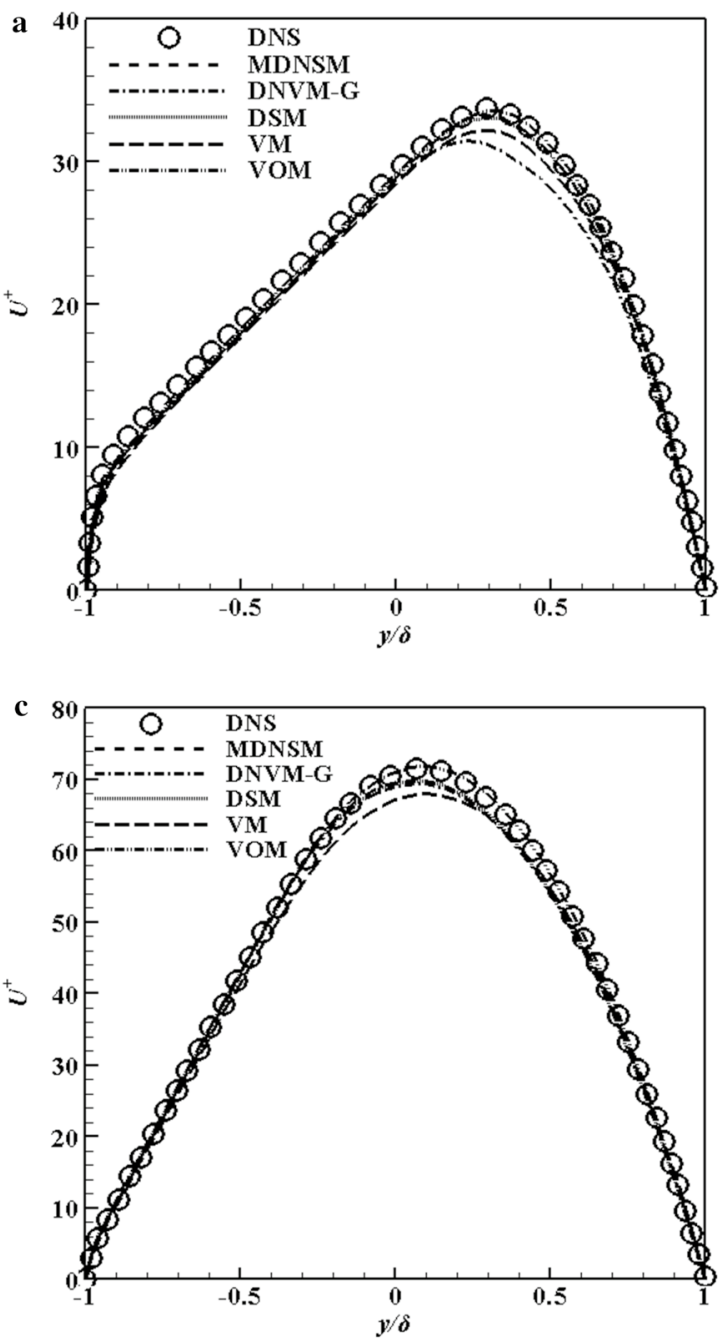

\section{Results}

Figure 2 shows the mean velocity profiles using different models in four different cases. From Fig. 2, we can see that the VOM model has a good agreement with the DNS result
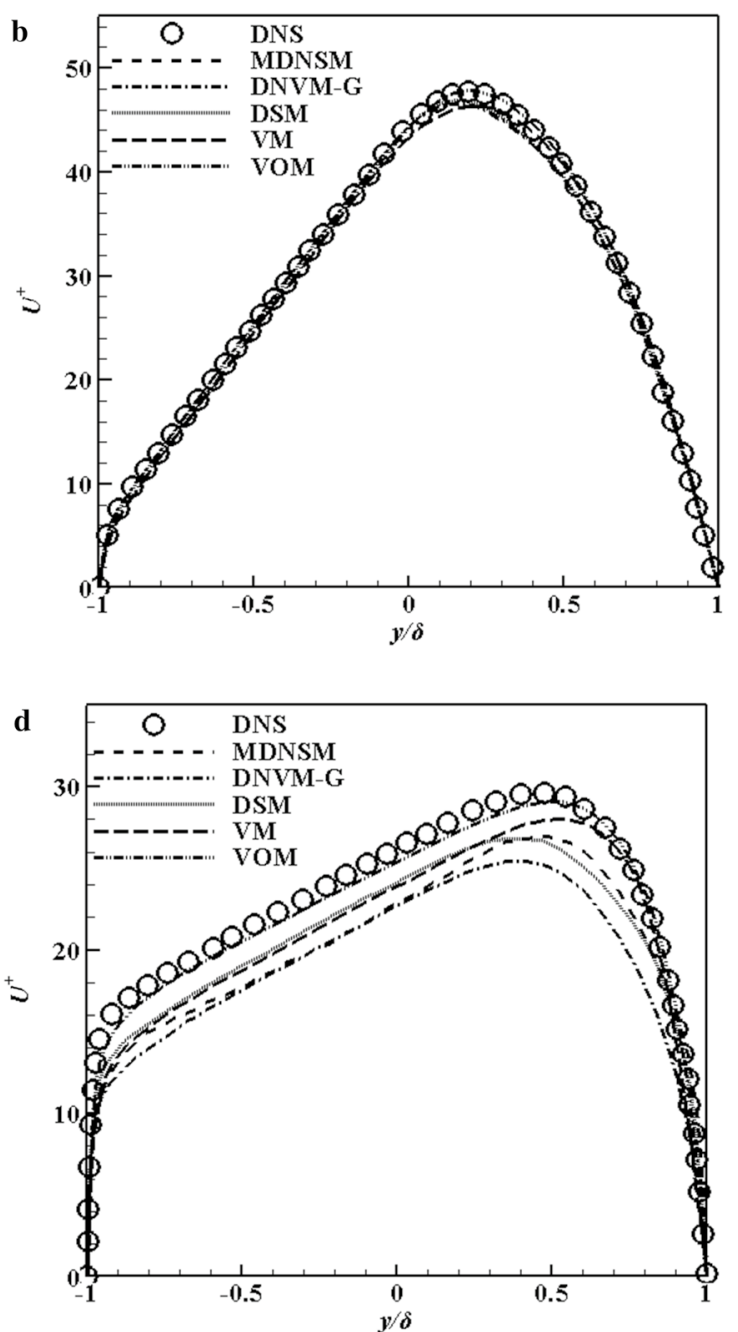

Fig. 2 Mean velocity profiles in different models: $\mathbf{a}$ at $R e_{\tau}=180, R o_{\tau}=22$, b at $R e_{\tau}=180, R o_{\tau}=40, \mathbf{c}$ at $R e_{\tau}=180, R o_{\tau}=80, \mathbf{d}$ at $R e_{\tau}=435$, $R o_{\tau}=10.3$ 

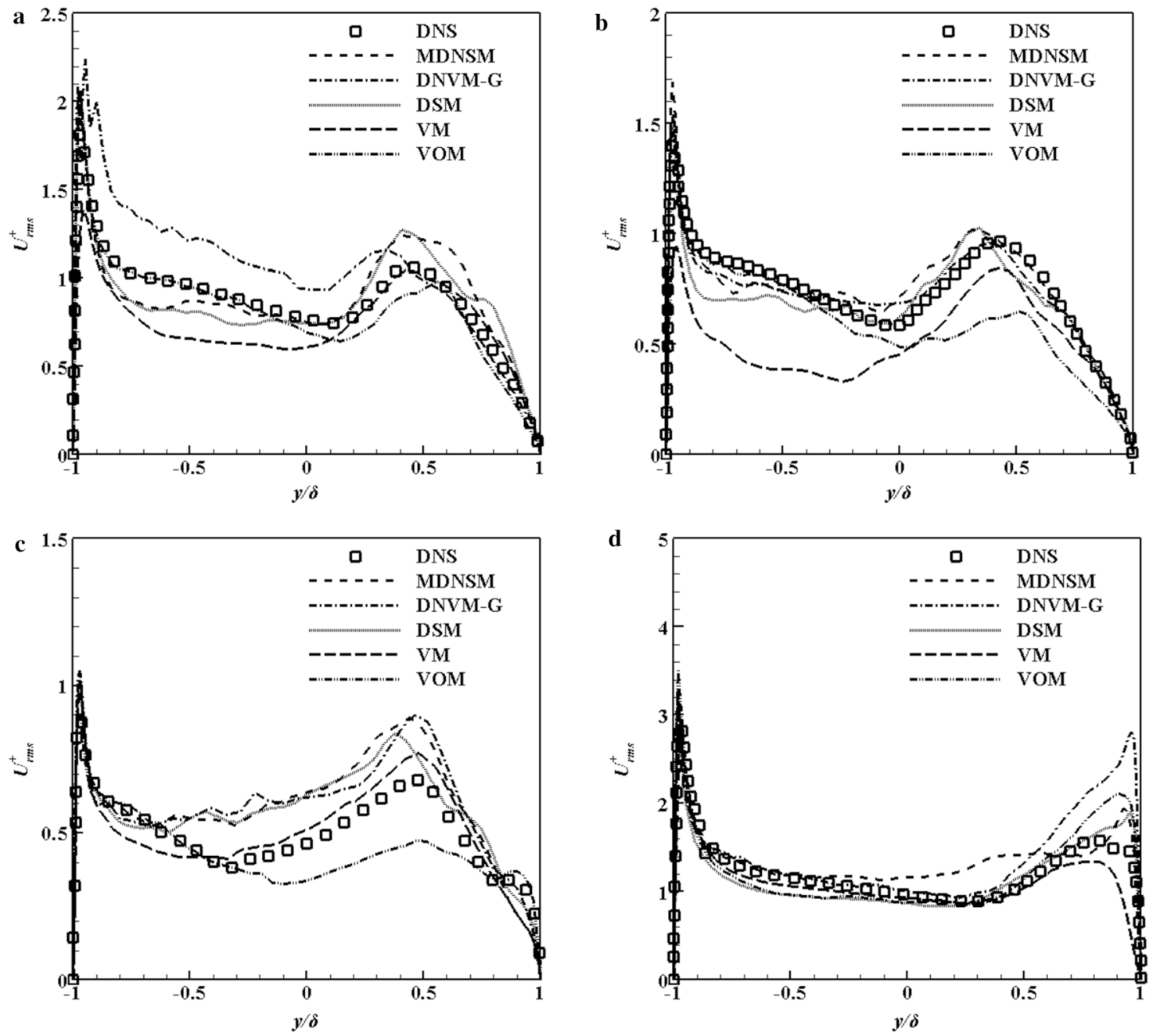

Fig. 3 Streamwise turbulence intensity in using different models: a at $R e_{\tau}=180, R o_{\tau}=22$, b at $R e_{\tau}=180, R o_{\tau}=40, \mathbf{c} R e_{\tau}=180, R o_{\tau}=80, \mathbf{d}$ at $R e_{\tau}=435, R o_{\tau}=10.3$

which is better than other models. As the Rossby number increases, the results of VOM still get better agreement with DNS. It suggests that the VOM is more effective with the increasing of the rotational influence.

In Fig. 3, we display the streamwise turbulence intensity by using different models in four different cases. As shown in Fig. 3a-d, VOM could better predict the streamwise turbulence intensity totally, especially on the pressure side than other models. In Fig. 3a, the VOM and DNVM-G models predict better than other models.

In Fig. 3b, the MDNSM and DNVM-G models predict better than other models. But at higher Rossby number, all of the models can't well predict the streamwise turbulence intensity of the suction side.

Figure 4 shows the results of the total Reynolds shear stress in different cases. The VOM model has a better result than other models. The MDNSM and DNVM-G models also get good results, but VM behaves worst among the models especially at high Rossby numbers. In Fig. 4d, near the suction side (especially at $y / \delta>0.7$ ), the VOM and MDNSM predict better than other models.

Figure 5 shows the averaged SGS eddy viscosity $\left\langle\nu_{\text {sgs }}\right\rangle$ (normalized by $\nu$ ) using different eddy-viscosity models. In Fig. 5, the averaged SGS eddy viscosity tends to 

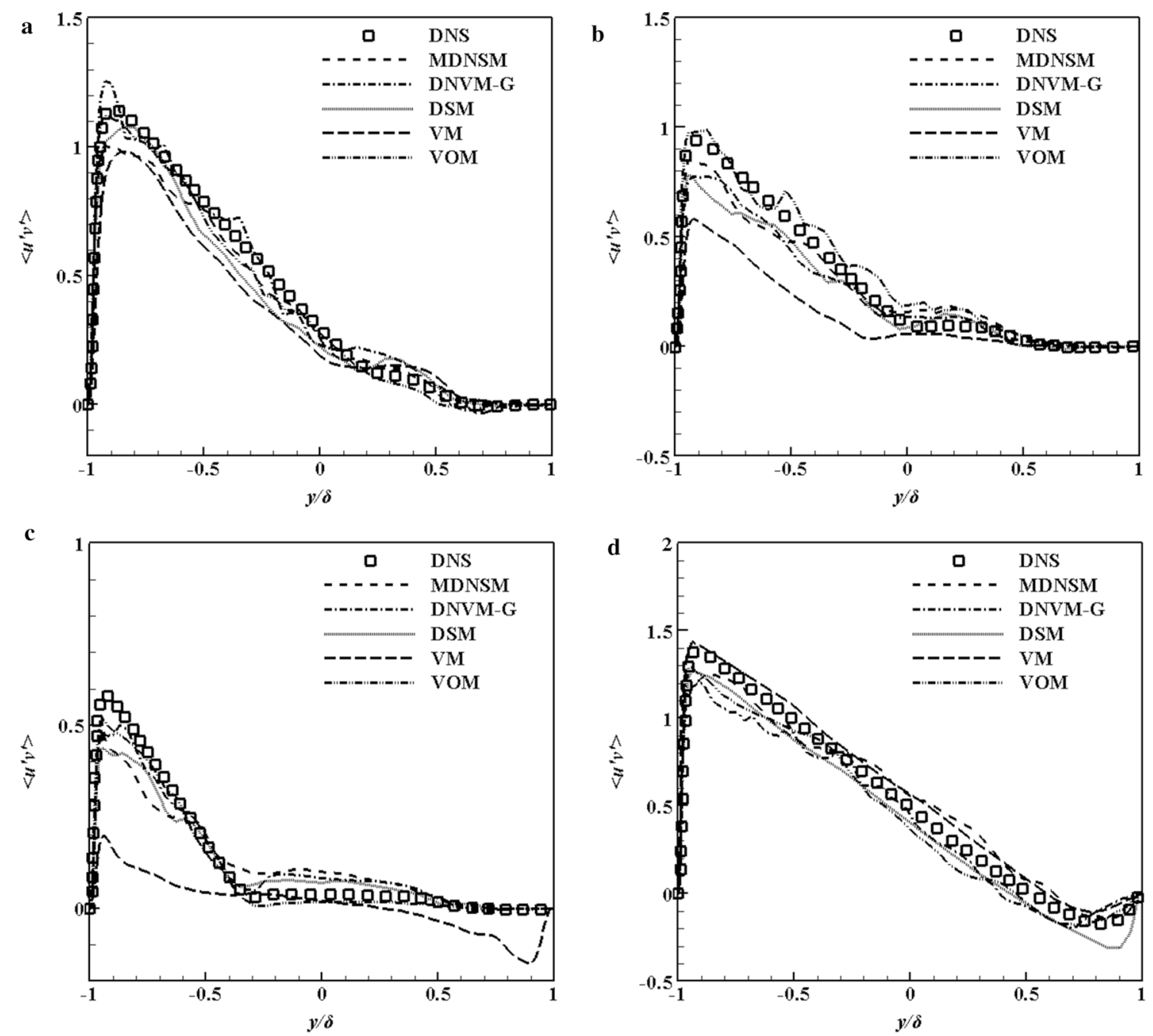

Fig. 4 Total Reynolds shear stress (the summation of resolved and modeled) using different models: $\mathbf{a}$ at $R e_{\tau}=180, R o_{\tau}=22$, $\mathbf{b}$ at $R e_{\tau}=180$, $R o_{\tau}=40, \mathbf{c} R e_{\tau}=180, R o_{\tau}=80, \mathbf{d}$ at $R e_{\tau}=435, R o_{\tau}=10.3$

zero near the suction side because of the suppression effect of the Coriolis force. In general, $<\nu_{s g s}>$ of the VOM is lower than $\left\langle\nu_{s g s}\right\rangle$ of the DSM especially at pressure side, so the dissipation produced by the VOM is lower than the DSM. Thus, in Figs. 3 and 4, the VOM gets a little higher results than the DSM and the results from the VOM have better agreement with DNS results.

Figure 6 shows the instantaneous isosurface of $Q$ from VOM at $R e_{\tau}=180, R o_{\tau}=22$. From Fig. 6, we can find the elongated large-scale vortex on the pressure (unstable) side. On another (suction) side, there are a few vortex structures and the flow tends to laminarization. And the vortex distribution in Fig. 6 reflects the main character in rotating wall turbulence.

The Taylor-Gortler vortices [25, 26] are important structures in rotating turbulent channel flows. It's significant for SGS models to well predict the Taylor-Gortler vortices. Figure 7 shows time-averaged Taylor-Gortler vortices on the $y-z$ plane from VOM. From Fig. 7, we can clearly observe three pairs of the large-scale roll cells. Thus the mean scale of one pair of the vortices is about $2 \pi h / 3$. 

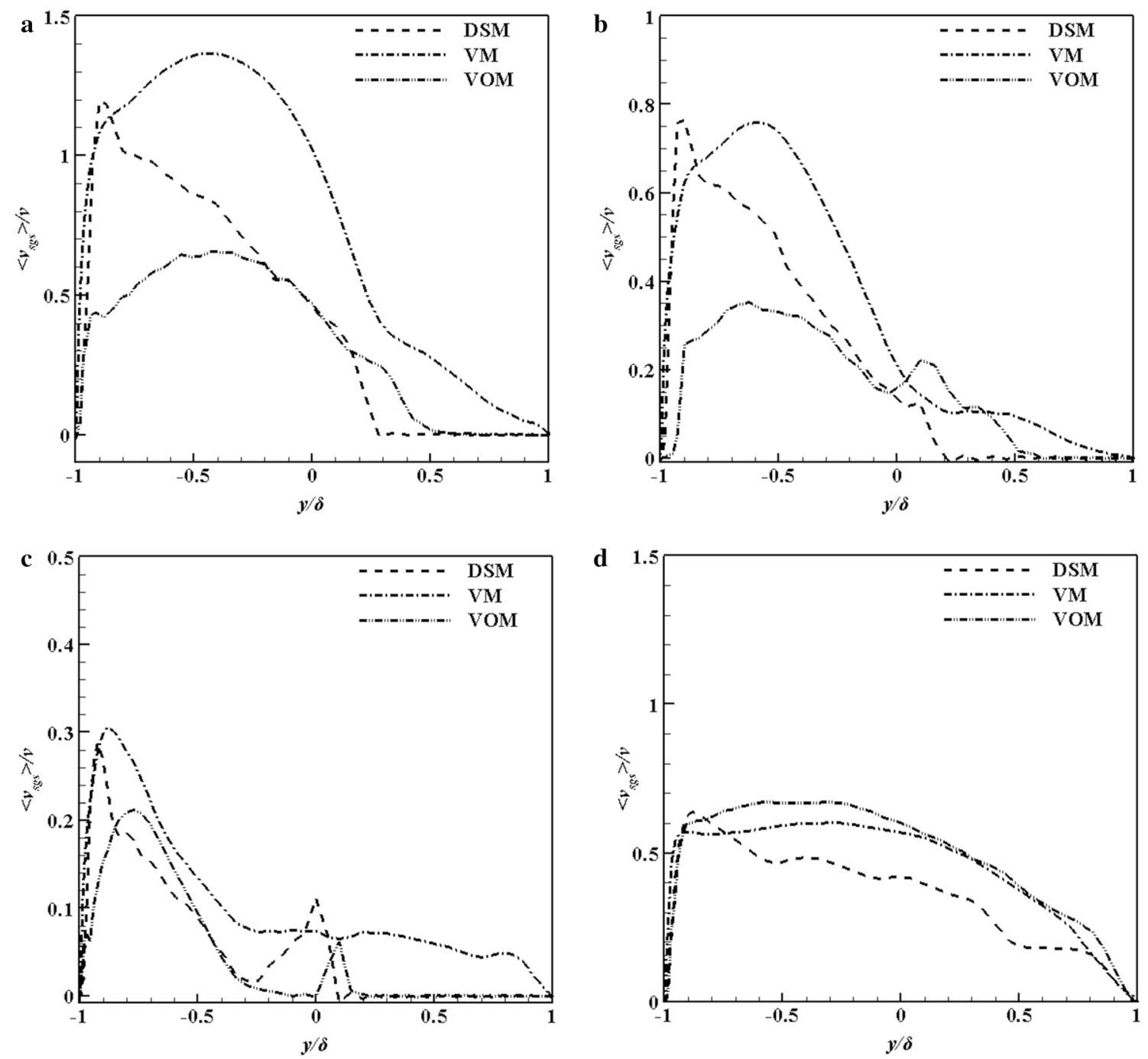

Fig. 5 Averaged SGS eddy viscosity $\left\langle\nu_{s g s}>\right.$ (normalised by $\nu$ ) in different viscosity models: $\mathbf{a}$ at $R e_{\tau}=180, R o_{\tau}=22, \mathbf{b}$ at $R e_{\tau}=180, R o_{\tau}=40, \mathbf{c}$ at $R e_{\tau}=180, R o_{\tau}=80, \mathbf{d}$ at $R e_{\tau}=435, R o_{\tau}=10.3$

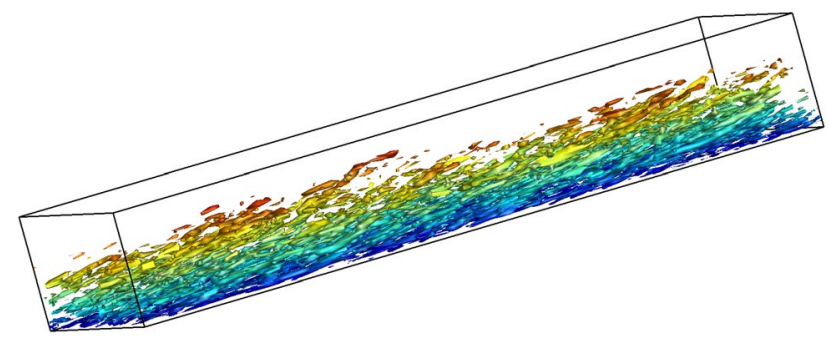

Fig. 6 Instantaneous isosurface of $Q$ (second invariant of the strain rate tensor) at $R e_{\tau}=180, R o_{\tau}=22$ using $\mathrm{VOM}$

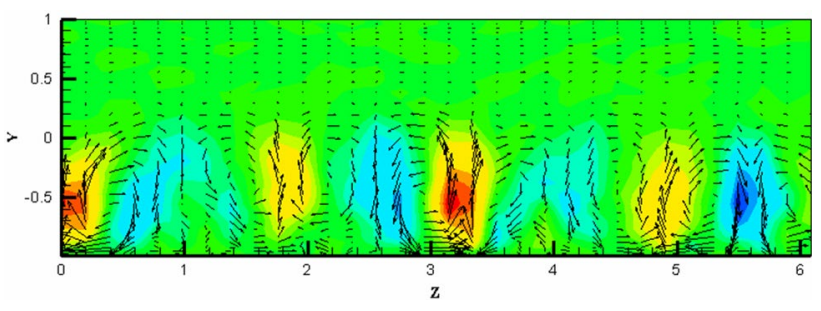

Fig. 7 Time-averaged Taylor-Gortler vortices on the $y-z$ plane from VOM 

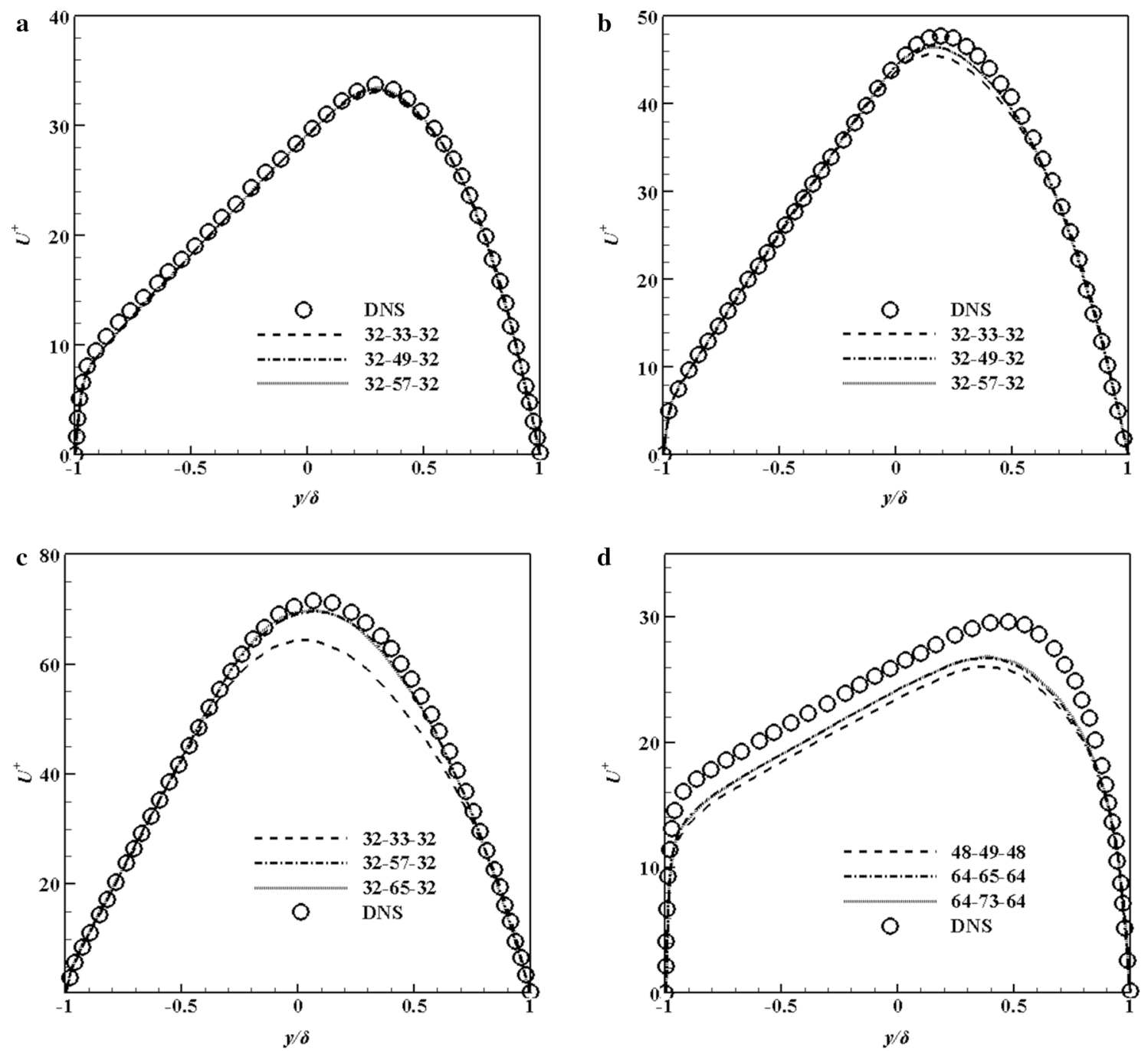

Fig. 8 Mean velocity profiles from different grid resolutions a at $R e_{\tau}=180, R o_{\tau}=22, \mathbf{b}$ at $R e_{\tau}=180, R o_{\tau}=40, \mathbf{c}$ at $R e_{\tau}=180, R o_{\tau}=80, \mathbf{d}$ at $R e_{\tau}=435, R o_{\tau}=10.3$

\section{Conclusion}

In this paper, based on the vorticity gradient tensor, and considering the proper scaling at the wall $\left(v_{s g s} \sim \mathrm{O}\left(y^{3}\right)\right)$, we construct a new wall-adaptive SGS model for rotating turbulent flows. Through analysis, we found that the vorticity gradient tensor plays a key role in rotating flows. The proposed model is tested in four different cases of spanwise rotating turbulent channel flows and the new model can obviously well predict the effect of rotation. Contrast to some often used SGS models, the VOM can obtain more precise simulating results, such as the mean velocity profiles, the streamwise turbulence intensity and total Reynolds shear stress, with the increase of rotational influence. At the same time, the new model can apparently predict the Taylor-Gortler vortices which is the main character vortex in rotating wall turbulent flows.

Overall, the VOM is a subgrid-scale model suitable for simulating rotating turbulence, and it needs no a second filtering, which will be easy to applied to the engineering rotational wall turbulence.

Acknowledgement This work was supported by the National Natural Science Foundation of China (Grants 91852203 and 11472278), the National Key Research and Development Program of China (Grant 2016YFA04-01200), Science Challenge Project (Grant TZ2016001), and Strategic Priority Research Program of Chinese Academy of Sciences (Grants XDA17030100 and XDC01000000). The authors thank the National Supercomputer Center in Tianjin (NSCC-TJ) and the National Supercomputer Center in Guangzhou (NSCC-GZ) for providing computer time. 


\section{Appendix: the grid independence}

We choose different grid resolutions for each case and then obtain a better grid resolution. $N_{x} \times N_{y} \times N_{z}=32 \times 33 \times 32$, $32 \times 49 \times 32,32 \times 57 \times 32$ are used in case $1\left(R e_{\tau}=180\right.$, $\left.R o_{\tau}=22\right), \quad N_{x} \times N_{y} \times N_{z}=32 \times 33 \times 32,32 \times 49 \times 32$, $32 \times 57 \times 32$ are used in case $2\left(R e_{\tau}=180, R o_{\tau}=40\right)$, $N_{x} \times N_{y} \times N_{z}=32 \times 33 \times 32,32 \times 57 \times 32,32 \times 65 \times 32$ are used in case $3\left(R e_{\tau}=180, R o_{\tau}=80\right)$, and $N_{x} \times N_{y} \times N_{z}=48 \times 49 \times 48$, $64 \times 65 \times 64,64 \times 73 \times 64$ are used in case $4\left(R e_{\tau}=435\right.$, $\left.R o_{\tau}=10.3\right)$.

In Fig. 8, we show the mean velocity profiles from different grid resolutions of different cases. From Fig. 8a, we found all the resolutions are close to each other. In Fig. 8b, the results from grid resolutions $32 \times 49 \times 32,32 \times 57 \times 32$ are close to the DNS result. In Fig. 8c, grid resolutions $32 \times 33 \times 32$ under-predicts the mean velocity profile, and the results of grid resolutions $32 \times 57 \times 32,32 \times 65 \times 32$ have a better agreement with the DNS result. In Fig. 8 d, the results of grid resolutions $64 \times 65 \times 64,64 \times 73 \times 64$ are close to each other and are higher than the results of grid resolution $48 \times 49 \times 48$. Thus, we use the grid resolution $32 \times 33 \times 32$, $32 \times 49 \times 32,32 \times 57 \times 32,64 \times 65 \times 64$ for case $1,2,3$, and 4 .

\section{References}

1. Smagorinsky, J.: General circulation experiments with the primitive equations: i the basic experiment. Mon. Weather Rev. 91, 99-164 (1963)

2. Deardorff, J.W.: A numerical study of three-dimensional turbulent channel flow at large reynolds numbers. J. Fluid Mech. 41, 453-480 (1970)

3. Chollet, J.P., Lesieur, M.: Parameterization of small scales of three-dimensional isotropic turbulence utilizing spectral closures. J. Atmos. Sci. 38(12), 2747-2757 (1981)

4. Cui, G.X., Xu, C.X., Fang, L., et al.: New subgrid eddy-viscosity model for large-eddy simulation of anisotropy turbulence. J. Fluid Mech. 582, 377-397 (2007)

5. Vreman, A.: An eddy-viscosity subgrid-scale model for turbulent shear flow: algebraic theory and applications. Phys. Fluids 16, 3670-3681 (2004)

6. Nicoud, F., Toda, H.B., Cabrit, O., et al.: Using singular valuesto build a subgrid-scale model for large-eddy simulations. Phys. Fluids 23, 085106 (2011)

7. Yu, C., Hong, R., Xiao, Z., et al.: Subgrid-scale eddy viscosity model for helical turbulence. Phys. Fluids 25(9), 095101 (2013)
8. Germano, M., Piomelli, U., Moin, P., et al.: A dynamic subgridscale eddy viscosity model. Phys. Fluids A 3(7), 1760-1765 (1991)

9. Lilly, D.K.: A proposed modification of the germano subgrid-scale closure method. Phys. Fluids A 4(3), 633-635 (1992)

10. Piomelli, U.: High reynolds number calculations using the dynamic subgrid-scale stress model. Phys. Fluids A 5(6), 1484 1490 (1993)

11. Meneveau, C., Lund, T.S., Cabot, W.H.: A lagrangian dynamic subgrid-scale model of turbulence. J. Fluid Mech. 319, 353-385 (1996)

12. Yu, C., Xiao, Z., Li, X.: Dynamic optimization methodology based on subgrid-scale dissipation for large-eddy simulation. Phys. Fluids 28(1), 015113 (2016)

13. Tafti, D.K., Vanka, S.P.: A numerical study of the effects of spanwise rotation on turbulent channel flow. Phys. Fluids A 3, 642-656 (1990)

14. Jiang, Z., Xia, Z., Shi, Y., et al.: Large-eddy simulation of spanwise rotating turbulent channel flow with dynamic variants of eddy viscosity model. Phys. Fluids 30(4), 040909 (2018)

15. Kobayashi, H.: The subgrid-scale models based on coherent structures for rotating homogeneous turbulence and turbulent channel flow. Phys. Fluids 17(4), 045104 (2005)

16. Yang, Z.X., Cui, G.X., Zhang, Z.S., et al.: A modified nonlinear sub-grid scale model for large-eddy simulation with application to rotating turbulent channel flows. Phys. Fluids 24(7), 075113 (2012)

17. Yang, Z.X., Cui, G.X., Xu, C.X., et al.: Large-eddy simulation of rotating turbulent channel flow with a new dynamic global-coefficient nonlinear subgrid stress model. J. Turbul. 13, N48 (2012)

18. Silvis, M.H., Verstappen, R.: Nonlinear subgrid-scale models for large-eddy simulation of rotating turbulent flows. In Direct and large-eddy simulation XI, pp. 129-134. Springer, Cham (2019)

19. Teitelbaum, T., Mininni, P.D.: Effect of helicity and rotation on the free decay of turbulent flows. Phys. Rev. Lett. 103(1), 014501 (2009)

20. Borue, V., Orszag, S.A.: Spectra in helical three-dimensional homogeneous isotropic turbulen-ce. Phys. Rev. 55, 7005 (1997)

21. Johnston, J.P., Halleen, R.M., Lezius, D.K.: Effects of spanwise rotationon the structure of two-dimensional fully developed turbulent channel flow. J. Fluid Mech. 56, 533-559 (1972)

22. Cale, B., Wang, B.C.: Direct numerical simulation of turbulent heat transfer in a wall-normal rotating channel flow. Int. J. Heat Fluid Flow 80, 108480 (2019)

23. Dai, Y.J., Huang, W.X., Xu, C.X.: Coherent structures in streamwise rotating channel flow. Phys. Fluids 31, 021204 (2019)

24. Cheng, Q., Ng, C.O.: Rotating electroosmotic flow of an Eyring fluid. Acta. Mech. Sin. 33(2), 295-315 (2017)

25. Yang, Z.X., Wang, B.C.: Capturing Taylor-Görtler vortices in a streamwise-rotating channel at very high rotation numbers. J. Fluid Mech. 838, 658-689 (2018)

26. Yang, Z.X., Deng, B.Q., Wang, B.C., et al.: The effects of streamwise system rotation on pressure fluctuations in a turbulent channel flow. Phys. Fluids 30, 091701 (2018) 